\title{
A NEW GENERALIZATION OF THE STURM COMPARISON THEOREM TO SELFADJOINT SYSTEMS
}

\author{
SHAIR AHMAD AND ALAN C. LAZER
}

\begin{abstract}
The Sturm Comparison Theorem is generalized to second order linear systems. It is based on a comparison of the elements of the matrices involved.
\end{abstract}

In this paper, we consider the vector differential equations

$$
y^{\prime \prime}+P(t) y=0
$$

and

$$
z^{\prime \prime}+Q(t) z=0
$$

where $P(t)=\left(p_{i j}(t)\right)$ and $Q(t)=\left(q_{i j}(t)\right)$ are continuous $n \times n$ symmetric matrices on a given interval $[a, b]$. For the case $n=1$ such equations have been studied extensively, beginning with the work of Sturm [7] in 1836. Since then there have been various extensions of the Sturmian theory to selfadjoint systems of second order linear differential equations, initiated by Morse [5] in 1930. Further extensions were subsequently given by Birkhoff and Hestenes, Reid, and others (see [6]). It was shown in [5] that if $P(t)$ and $Q(t)$ are symmetric, $Q(t) \geqslant P(t)$, i.e. $Q(t)-P(t)$ is positive semidefinite, with $Q(\bar{t})$ $>P(\bar{t})$ for some number $\bar{t}$ in the interval $[a, b]$, and if (1) has a nontrivial solution $y(t)$ satisfying $y(a)=y(b)=0$, then (2) has a nontrivial solution $z(t)$ such that $z(a)=z(c)=0$, where $c$ is some number in the open interval $(a, b)$. The purpose of this note is to present a similar theorem which is based on an elementwise comparison of the matrices $P(t)$ and $Q(t)$. Our theorem neither implies the theorem of Morse nor is it implied by it. We are able to give a relatively simple proof based on variational methods and an earlier result of ours.

We recall that a number $b, b>a$, is said to be conjugate to a relative to a certain equation of the form (1) if there exists a nontrivial solution of this equation which vanishes at $a$ and $b$. The equation is said to be disconjugate on an interval $I$ if no nontrivial solution of it vanishes more than once in $I$. It is well-known that in the selfadjoint case, i.e. if $P(t)$ is symmetric, conjugate points are isolated. Let $\mathbb{Q}[a, b]$ denote the set of absolutely continuous $R^{n}$-valued functions $h(t)$ on $[a, b]$ such that $\left|h^{\prime}\right| \in L^{2}[a, b]$ and $h(a)=h(b)$

Received by the editors October 28, 1976.

AMS (MOS) subject classifications (1970). Primary 34A25; Secondary 34C10.

Key words and phrases. Conjugate point, disconjugate, symmetric, selfadjoint, solution. 
$=0$. The proof of the following lemma, which essentially follows from Reid [6, p. 332], has been given in [2]. For more information on the preceding concepts one might consult [4] and [6].

LEMMA 1. Let $A(t)$ be a continuous $n \times n$ symmetric matrix on $[a, b]$, and let $J[h ; a, b]$ denote the functional

$$
J[h ; a, b]=\int_{a}^{b}\left(\left\langle h^{\prime}, h^{\prime}\right\rangle-\langle A h, h\rangle\right) d t
$$

over the set $\mathbb{Q}[a, b]$ of admissible functions. Then $J[h ; a, b] \geqslant 0$ for all $h$ in $\mathbb{Q}[a, b]$ if and only if the interval $[a, b]$ contains no point conjugate to $a$ in its interior relative to the equation $x^{\prime \prime}+A(t) x=0$.

REMARK 1. The above lemma is a slight modification of the well-known fact (see, e.g., [3] or [6]) that the equation

$$
x^{\prime \prime}+A(t) x=0
$$

is disconjugate on the interval $[a, b]$ if and only if

$$
\left.J[h ; a, b]=\int_{a}^{b}\left(\left\langle h^{\prime}, h^{\prime}\right\rangle-\langle A h, h\rangle\right) d t\right\rangle 0
$$

over the set of admissible functions $h, h \neq 0$.

Theorem 1. Assume that in equations (1) and (2), $q_{i j}(t) \geqslant p_{i j}(t)$ for $1 \leqslant i$, $j \leqslant n$, and $t \in[a, b]$. Further, assume that $q_{i i}(\bar{t})>p_{i i}(\bar{t})$ for some $\bar{t} \in(a, b)$, $1 \leqslant i \leqslant n$, and that $p_{i j}(t) \geqslant 0$ for $i \neq j$. If (1) has a nontrivial solution $y(t)$ satisfying $y(a)=y(b)=0$, then (2) has a nontrivial solution $z(t)$ such that $z(a)=z(c)=0, a<c<b$.

We note that in the above theorem only the diagonal elements of $Q$ are required to be strictly greater than those of $P$ at a point $\bar{t}$. It can be verified, by letting $P(t)=\operatorname{diag}(0,1)$ and $Q(t)=\operatorname{diag}(1,1)$, that this condition cannot be relaxed any further.

Proof of Theorem 1. First we assume that $b$ is the first conjugate point of $a$ relative to (1). By Theorem 1 of [1] (or Theorem 2 of [2]) it follows that there exists a nontrivial solution

$$
u(t)=\operatorname{col}\left(u_{1}, \ldots, u_{n}\right)
$$

of (1) such that $u(a)=u(b)=0$ and $u_{j}(t) \geqslant 0$ on $[a, b], j=1, \ldots, n$. Let $J[h ; a, b]$ and $\tilde{J}[h ; a, b]$ define the functionals given by

$$
J[h ; a, b]=\int_{a}^{b}\left(\left\langle h^{\prime}, h^{\prime}\right\rangle-\langle P h, h\rangle\right) d t
$$

and

$$
\tilde{J}[h ; a, b]=\int_{a}^{b}\left(\left\langle h^{\prime}, h^{\prime}\right\rangle-\langle Q h, h\rangle\right) d t
$$

over the set $\mathbb{Q}[a, b]$ of admissible functions. Then, 


$$
\begin{aligned}
\tilde{J}[u ; a, b] & =\int_{a}^{b}\left(\left\langle u^{\prime}, u^{\prime}\right\rangle-\langle Q u, u\rangle\right) d t \\
& =\int_{a}^{b}\left(\left\langle u^{\prime}, u^{\prime}\right\rangle-\sum_{i=1}^{n} q_{i i}(t) u_{i}^{2}-\sum_{i \neq j} q_{i j}(t) u_{i} u_{j}\right) d t \\
& <\int_{a}^{b}\left(\left\langle u^{\prime}, u^{\prime}\right\rangle-\sum_{i=1}^{n} p_{i i}(t) u_{i}^{2}-\sum_{i \neq j} p_{i j} u_{i} u_{j}\right) d t \\
& =\int_{a}^{b}\left(\left\langle u^{\prime}, u^{\prime}\right\rangle-\langle P(t) u, u\rangle\right) d t=J[u ; a, b] .
\end{aligned}
$$

The above inequality follows from the hypothesis that $p_{i i}(\bar{t})<q_{i i}(\bar{t}), 1 \leqslant i \leqslant$ $n$. Multiplying the equation

$$
u^{\prime \prime}+P(t) u=0
$$

by $-u$ and integrating from $a$ to $b$ we see that

$$
\int_{a}^{b}\left(\left\langle u^{\prime}, u^{\prime}\right\rangle-\langle P u, u\rangle\right) d t=0
$$

We have thus shown that $\tilde{J}[u ; a, b]<J[u ; a, b]=0$. By Lemma $1 a$ has a conjugate point $c$ in the open interval $(a, b)$ relative to equation (2). Consequently, there exists a nontrivial solution $z(t)$ of (2) satisfying $z(a)=$ $z(c)=0$, where $c$ is some number in the open interval $(a, b)$.

Now, let us assume that $b$ is not the first conjugate point of $a$ relative to (1). Let $\eta(a)$ be the first conjugate point of $a$, and let

$$
v(t)=\operatorname{col}\left(v_{1}, \ldots, v_{n}\right)
$$

be a solution of (1) such that $v(a)=v(\eta(a))=0$ and $v_{j}(t) \geqslant 0, j=1, \ldots, n$. Then $a<\eta(a)<b$. The same argument that we gave to establish that $\tilde{J}[u ; a, b]<J[u, a, b]$ shows that

$$
\tilde{J}[v ; a, \eta(a)] \leqslant J[v ; a, \eta(a)] .
$$

We no longer have strict inequality since wè can not assume that $\bar{t} \in$ $[a, \eta(a)]$. However, $\tilde{J}[v ; a, \eta(a)] \leqslant J[v ; a, \eta(a)]=0$ implies (see Remark 1) that (2) is not disconjugate on $[a, \eta(a)]$. Hence the interval $[a, \eta(a)]$ contains a point conjugate to $a$ relative to (2). For, it follows (see [3] or [6]) that if $\tilde{\eta}(a)$ is the first conjugate point of $a$ relative to (2) then (2) is disconjugate on $[a, \tilde{\eta}(a))$. Hence we must have $\tilde{\eta}(a) \leqslant \eta(a)$. This shows that (2) has a nontrivial solution $z(t)$ such that $z(a)=z(c)=0$, where $c$ is some number in the interval $(a, \eta(a)]$, and the proof is complete.

\section{REFERENCES}

1. S. Ahmad and A. C. Lazer, Component properties of second order linear systems, Bull. Amer. Math. Soc. 82 (1976), 287-289.

2. $\quad$ On the components of extremal solutions of second order systems, SIAM J. Math. Anal. 8 (1977), 16-23.

3. W. A. Coppel, Disconjugacy, Lecture Notes in Math., vol. 220, Springer-Verlag, Berlin and New York, 1971. 
4. I. M. Gelfand and S. V. Fomin, Calculus of variations, Prentice-Hall, Englewood Cliffs, N. J., 1963.

5. M. Morse, A generalization of the Sturm separation and comparison theorems in n-space, Math. Ann. 103 (1930), 72-90.

6. W. T. Reid, Ordinary differential equations, Wiley, New York, 1971.

7. J. C. F. Sturm, Mémoire sur les équations différentielles linéaires de second ordre, J. Math. Pures Appl. 1 (1836), 106-186.

Department of Mathematics, Okiahoma State University, Stillwater, OkLahoma 74074

Department of Mathematics, University of Cincinnati, Cincinnati, Oho 45221 